\title{
Varying the componentwise order of the multistep methods in solving ODEs and its absolute stability
}

\begin{abstract}
Most variable order, variable stepsize codes for solving ODEs vary the order along the interval of integration but keep the order constant across the system. However, some codes that do partitioning of the ODEs into stiff and nonstiff subsystems vary the order for each equation in the system. In this paper, the reasons for varying the order componentwise are given and some of its salient features are illustrated using a numerical example. Also given is the direct proof of the existence of a region of absolute stability when different order Adams explicit methods are used to solve a system of ODEs.
\end{abstract}

Keyword: ODEs; Componentwise 